\title{
Multifunctional Room Controller Design
}

\author{
Tang Lili, a , Huang Wei ${ }^{2, b}$ \\ ${ }^{1}$ Department of Computer \& Information Engineering, Zhixing College of Hubei University, Wuhan, \\ 430011, China \\ ${ }^{2}$ System Design Institute of Hubei Aerospace Technology Academy, Wuhan, 430040, China \\ aemail: toney2001@126.com, bemail:jijunhuang@126.com
}

Keywords: environmental monitoring; MCU AT89S52; digital sensor

\begin{abstract}
This paper is mainly to study the working state of a certain space appliance through the MCU and the circuit. The main core of the design is composed by the control core microcontroller AT89S52, digital sensor, LCD FM12864M-12L and some other peripheral circuits. This paper mainly introduces the performance, inner structure, input and output structure of the AT89S52. It shows the driving mode of the DS1302, the DS18B20, the DHT11, and digital sensors such as the pyroelectric infrared, which are all the products of the DALLAS company. This design depends on the collections of the various environmental indicators by all the sensors mentioned above and its sending the collected data to the MCU. The MCU will set up the system parameter through the keys according to the various environmental indicators and the user, driving relay and a buzzer to control the operation of each electrical appliance and give the alarm. It finally designs human-computer interactive multifunctional controller interface based on LCD FM12864M-12L.
\end{abstract}

\section{Introduction}

With the development of electronic technology, the function of today's electronic products is increasingly various and the sales price is lower, so its access to people's home life becomes more. As a result, it also brings many difficulties in the management of these household electrical appliances. And with people's improving intelligent requirements at home or in office, the need of a controller to supervise appliances effectively is more urgent. The controller enables efficient operation of more energy-saving and intelligent, also it let people live more comfortable and work more efficient, so as to obtain higher social and economic benefits.

The designed multifunctional room controller use the measurement unit composed by various digital sensor to measure environmental indicators, and then transmit the measured results to the MCU through a variety of bus protocol. At the same time, the control core will output all system operating parameters and environment index to the LCD 12864 screen real-time. The user can set up the system through the keyboard. At last, MCU will operate all kinds of electrical appliances in the room according to user keyboard input and a variety of environmental monitoring index, and will output various alarms and reminders to the LCD 12864 and buzzer. This design mainly has the following functions: (1) the calendar and clock function; (2) alarm function; (3) a friendly human-computer interaction interface; (4) human activity induction in the room; (5) environmental humidity measurement; (6) environmental temperature measurement; (7)Bluetooth data transmission. The design is based on the control core microcontroller AT89S52, other digital sensors and graphic LCD, which can control all kinds of electrical appliances in the room according to user settings and environmental indicators. It can not only provide users with convenient control experience, but also a more reasonable application of energy, so that people can live more comfortable and environmentally friendly.

\section{Working Principle of Controller}

According to the functional and hardware components, the framework of the design plan is as shown in the following diagram. 


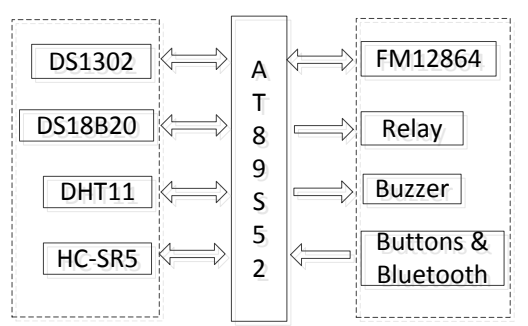

Fig.1. System hardware block diagram

As shown, the design discussed in this paper divide the whole system into four mainly sub modules according to the similar and related characteristics of the various functional blocks. They are microprocessor control sub module, sensor sub module which converts non electricity into electricity, display output user interactive sub module and user input sub module.

The design use A89S52 as the main control core to coordinate communication between the various modules, control all modules and make them work effectively. It is also the core device of the processor sub module The sub module of the sensor is mainly composed of core chips such as DS1302, DS18B2, DHT11, HC-SR5 etc., which is respectively clock chip, temperature sensor, temperature and humidity sensors, human thermal release electric sensor. The main function of the sensor and chip is collecting non electrical signals in the environment, and then convert it into electric signal. Also according to the internal functional circuit, it processes these electric signals and makes it be able to be recognized and processed by single chip microcomputer, then it is transmitted to MCU [1] through particular bus. In the output display module, graphic LCD FM12864-12L and buzzer is used to display and output the running state and parameter real-time, and output some cue tones and alarm signals, thus offer a good interface for the user interaction. In the user input sub module, two input methods are used, which are respectively the independent key composed of buttons, and Bluetooth transmission input composed of Bluetooth chip HC-06. Hardware and software design of the clock module.

\section{Design of control center and surrounding modules}

\section{Design of the clock module}

DS1302 is a trickle charging clock chip launched by DALLAS company. It contains a real-time clock / calendar and a static RAM of 31 bytes, which communicates with the microcontroller through a simple serial interface. Real-time clock / calendar circuit provides information of second, minute, hour, day, date, month and year. The clock chip communicates with the microcontroller in a serial mode through the RES, I/O and SCLK data lines.

In the program design, the user can adjust the time of the current system by using a button, and then write the modified data into DS1302. It also provides alarm clock function. Users can set the alarm clock through buttons, and can choose to turn off the alarm clock or open the alarm clock. When the set time of the alarm clock arrives, the buzzer will end a warning tone to the user. The circuit design is as follows.

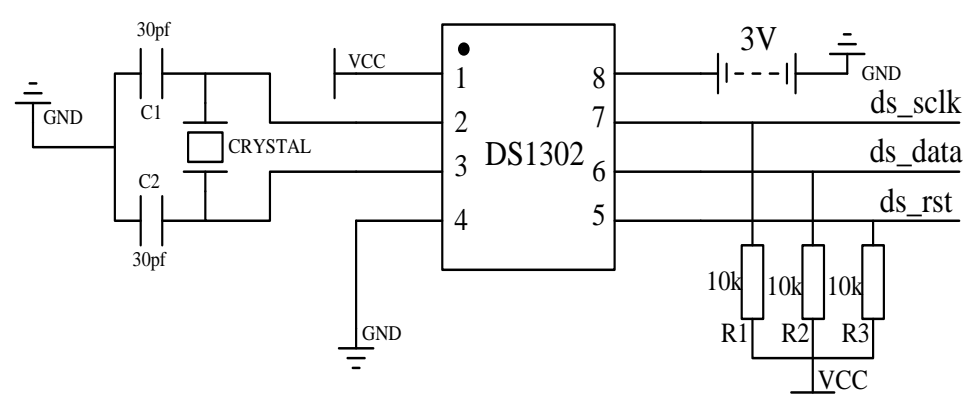

Fig.2. Clock circuit diagram 


\section{Design of temperature and humidity sensor}

\section{Circuit design of temperature sensor}

DS18B20 is a single line digital temperature sensor. When it connects with the microprocessor using a single bus interface mode, only a port line is needed to realize two-way communication between microprocessor and DS18B20.It has high anti-jamming ability, which makes it able to work in harsh industrial environments. Also it has a very wide temperature range of - 55 DEG C $\sim+125$ DEG C, and relatively high accuracy. In this design, it is used as a main temperature measuring device.

In order to communicate normally with MCU, read and write sequence provided by the manufacturer must be strictly followed in driver design. In the part of software design, the current temperature will be displayed real-time in the main interface, and also the setting function of temperature alarm is provided. The user can input a temperature interval through the button, thus when the temperature exceeds the set range, the buzzer will alarm [2]. Design circuits are as follows.

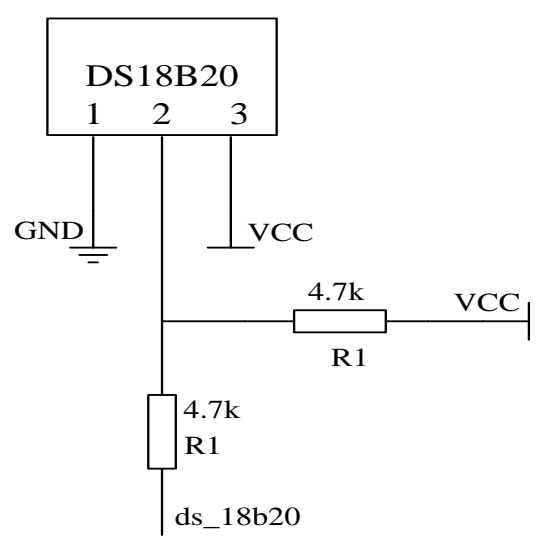

Fig.3. Temperature sensing circuit diagram

\section{Circuit design of humidity sensor}

DHT11 is a temperature and humidity complex sensor which contains calibrated digital signal output. It uses special digital module acquisition and temperature and humidity sensing technology, which ensures high reliability and excellent long-term stability of the product. Circuit design is as follows.

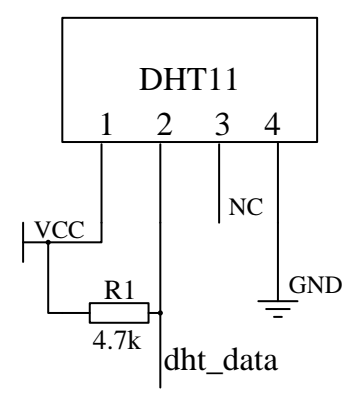

Fig.4. DHT11 connection diagram

\section{Detection design of human activity in the space}

Detecting whether there is human activity in a certain space can realize intelligent operations. In this design, relating to the liquid crystal according to the detection results can realize turning on the LCD backlight when there is human activity in a certain space and on the contrary turning off it when there is no human activity. Of course, the relevant user can choose whether to relate or not

HC-SR501 is automatic control module based on infrared technology, using imported probe 
LHI778 design from Germany, which has high sensitivity, strong reliability, ultra low voltage working mode and it is widely applied in many kinds of automatic induction electrical equipment, especially in automatic control products powered with dry battery, human activities within 7 meters can be detected. And the output will delay.

\section{LCD module design}

In this design, the modules are numerous. Comprehensive consideration of Single chip microcomputer resources under the premise of reasonable use, graphic LCD FM12864-12L is chosen as the output vector of the image interface.

LCD FM12864-12L owns font. Driver only needs to write the corresponding character code into the memory, and specify the location where you want to display on the screen, the right display on the screen can be obtained. As for LCD reading and writing, normal communication can be proceed as long as sketch in strict accordance with the read and write timing provided by manufacturers. Also special attention should be paid to not forget to detect LCD busy signal, and only in the non busy state can LCD be operated.

\section{The design of buzzer and relay}

Because the active buzzer does not require the user to prepare a specific audio encoding, users only need to give a high level, the buzzer will output a fixed frequency of audio signal. Drivers are more convenient, so the selection is active buzzer Relay is as the main control output device in the design. MCU can control the opening and closing of the relays through the output high and low level, so as to achieve the purpose of controlling electric appliance. Special attention should be paid to the relay as the inductive device in the hardware circuit design of the relay. Its opening and closing will produce impulse current and voltage, which affect the stable operation of SCM. The SCM can't directly drive it, so there should be an isolation link between them. Optocoupler isolation is used in this design [3].

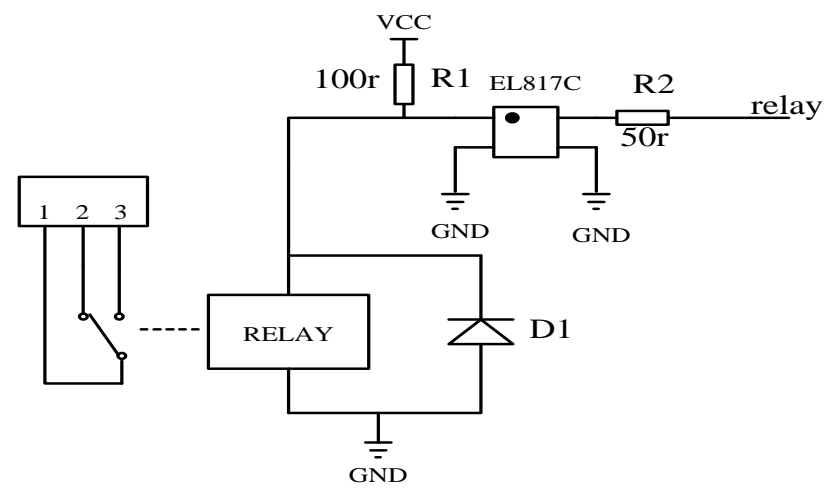

Fig.5. Relay output circuit

\section{Design of dental penetration module and button}

The Bluetooth transmission module adopts HC-06, which has the characteristics of stable transmission, simple interface, easy operation and so on. In this design, Bluetooth module and MCU are connected by serial communication mode, the default baud rate of which is 9600 . An intelligent mobile phone with a Bluetooth serial port is used in the input of the user side. Characters with a specific code can be sent through the application just by downloading and installing a Bluetooth serial port tools on the phone. Then SCM receives and analyzes it and treat it as the same as the button input.

In this design, the keyboard is the main input mode. There are eight buttons, four of which form a set of direction keys, and the other four are enter setup, exit settings, confirm and relay fast operation. 


\section{Software design of multifunctional room controller}

Compared to hardware, software design is more complex. In this design, the hardware is mostly digital logic device, such as digital sensor, LCM and so on, so there is no complex circuit design. But the software driver of these digital devices is more complex, which is due to different digital devices with different data interfaces, which needs to be driven by different software program. And software is indispensable in the realization of various functions, especially in dealing with the user keys, the software design has a very complex logic structure and complicated programming [4] [5].

In software design, the following function can be achieved: display real-time calendar, clock, temperature and humidity in the main interface; read the user's key input; set the system parameters according to user's input; complete alarm clock function by judging whether the current system time is the setting value of the alarm; to judge whether the current temperature exceeds the set alarm value and output the corresponding major functions such as tips.

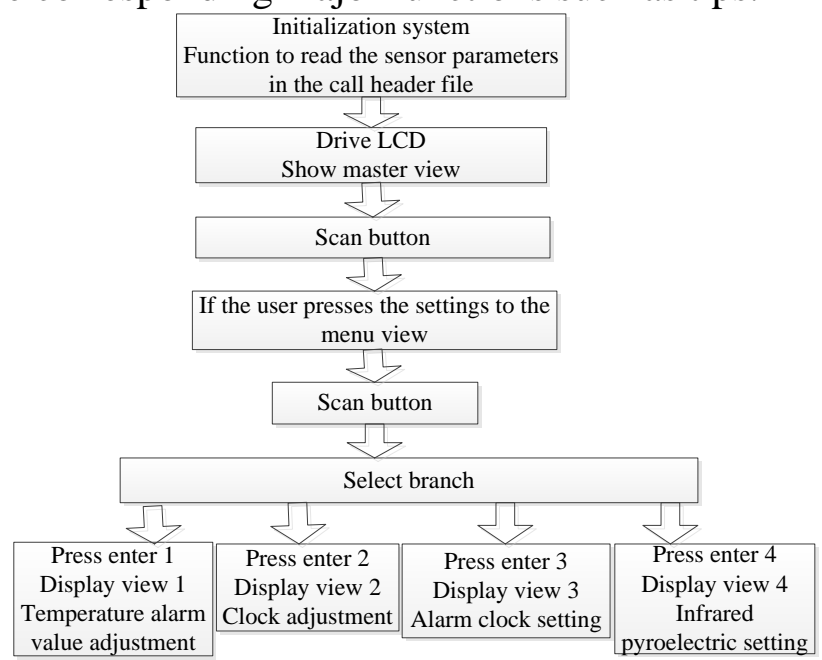

Fig.6. Software structure diagram

\section{Conclusion}

The acquisition and modification of system time in the design, the collection of environmental temperature and humidity, inducing whether there is human activity in a certain space and eventually displaying these parameters in the LCD, and system's making the corresponding processing action to the device according to the environmental parameters successfully complete the basic function design of the room controller. With the further improvement of modern electrification, this design is believed to have a big development space and a broad market prospect.

\section{Acknowledgement}

In this paper, the research was sponsored by the Science and Technology Research Program of 2015 Hubei Provincial Education Department (Project No. B2015514)

\section{Reference}

[1] Yu Zhigen. Sensor and detection technology (Second Edition) [M]. Beijing: Science Press, 2004.

[2] Wang Furui. Design of microcomputer measurement and control system based on single chip microcomputer [M]. Beijing: Beijing Aerospace University Press, 1999.

[3] Li Guangdi. The basis of single chip microcomputer [M]. Beijing: Beijing Aerospace University Press, 2001.

[4] Tan Haoqiang. C language programming (Third Edition) [M]. Beijing: Tsinghua University Press, 2005. 
[5] Chen Haiyan. 51 single chip microcomputer principle and application - based on C Keil and Proteus[M]. Beijing: Beijing Aerospace University Press, 2010. 